Cahiers
de la Recherche
sur les Droits

Cahiers de la recherche sur les droits

Fondamentaux fondamentaux

$18 \mid 2020$

La vulnérabilité

\title{
Vulnérabilité et sociétés
}

Aïda Bennini

\section{OpenEdition}

Journals

Édition électronique

URL : https://journals.openedition.org/crdf/6452

DOI : $10.4000 /$ crdf.6452

ISSN : 2264-1246

Éditeur

Presses universitaires de Caen

Édition imprimée

Date de publication : 19 novembre 2020

Pagination : 93-99

ISBN : 978-2-84133-987-7

ISSN : 1634-8842

Référence électronique

Aïda Bennini, «Vulnérabilité et sociétés », Cahiers de la recherche sur les droits fondamentaux [En ligne], 18 | 2020, mis en ligne le 19 novembre 2021, consulté le 14 novembre 2022. URL : http:// journals.openedition.org/crdf/6452 ; DOI : https://doi.org/10.4000/crdf.6452 


\title{
Vulnérabilité et sociétés
}

\author{
Aïda BENNINI \\ Maître de conférences en droit privé à l'université de Caen Normandie \\ Institut Demolombe (EA 967)
}

I. La vulnérabilité de la société à l'égard de ses organes

A. La vulnérabilité de la société à l'égard de ses associés

B. La vulnérabilité de la société à l'égard de ses dirigeants

II. La vulnérabilité de la société à l'égard des tiers

A. Les vulnérabilités de la société à l'égard de ses cocontractants

B. Les vulnérabilités de la société à l'égard de ses parties prenantes

La vulnérabilité est un état de fait rendant la personne qu'il atteint faible ou fragile. Le droit reconnaît, à travers plusieurs de ses sous-disciplines, la vulnérabilité des personnes afin de leur apporter une protection dans leur autonomie, leur intégrité physique, mentale, et leur dignité. Cette protection juridique des vulnérables puise son fondement dans l'ordre public de protection qui exprime l'idée selon laquelle le droit est l'instrument de la justice, et qu'il n'y a pas de justice sans équilibre.

La société, en tant que structure juridique de l'activité économique, n'échappe pas à l'ordre public de protection à l'instar d'autres contrats ${ }^{1}$. Ces dernières années, le droit des sociétés a considérablement évolué dans le sens d'une meilleure protection des actionnaires minoritaires ${ }^{2}$. Mais la société elle-même peut être vulnérable en raison d'imperfections organisationnelles ou conjoncturelles. L'imperfection organisationnelle est synonyme de facteurs intrinsèques à la société tandis que l'imperfection conjoncturelle visera les facteurs extrinsèques à la société.
Quelle qu'en soit sa cause, la vulnérabilité de la société est un état de fait qui aura pour conséquence directe ou indirecte de l'empêcher d'exercer normalement ses droits et/ ou ses prérogatives. Au regard des attentes légitimes découlant du contrat de société, la vulnérabilité pourrait correspondre à un état qui menace la réalisation de l'intérêt social. Étant rappelé que la légitimité s'apprécie par rapport à la finalité du droit, de l'institution ou du contrat dans lequel l'attente s'insère 3 .

Au-delà de l'évolution du droit, la question de la vulnérabilité des sociétés est régulièrement posée dans le débat public. Elle est même au cour de la politique économique dans une époque où les sociétés sont devenues perméables aux aléas extérieurs de toute nature, quelle que soit leur taille, en raison d'une globalisation circulaire. Les maux des unes deviennent alors ceux des autres, et les mécanismes juridiques tentent d'apporter une partie de la solution prônée par la politique économique poursuivie. Ces temps de crise sanitaire qui secouent le

1. Voir notamment J. Calais-Auloy, «Droit du marché et droit commun des obligations. L'influence du droit de la consommation sur le droit des contrats", Revue trimestrielle de droit commercial, 1998, p. 115 sq.; J. Mestre, "L'ordre public dans les relations économiques ", in L'ordre public à la fin du XX $X^{e}$ siècle, T. Revet (dir.), Paris, Dalloz, 1996, p. 33 sq.; Y. Strickler, «La protection de la partie faible en droit civil», Les petites affiches, $\mathrm{n}^{\circ} 213,25$ octobre 2004 , p. 6-9.

2. C. Hannoun, «L'équilibre des pouvoirs au sein des sociétés par actions", in Le contrôle des entreprises. Évolution et perspectives, B. Le Bars, C. Hannoun (dir.), Paris, L'Harmattan, 2007, p. 15 sq.

3. À propos de la notion d'attente légitime, voir G. Wicker, Les fictions juridiques. Contribution à l'analyse de l'acte juridique, préface de J. AmielDonat, Paris, LGDJ, 1997, p. 52 sq.; J. Valiergue, Les conflits d'intérêts en droit privé. Contribution à la théorie juridique du pouvoir, préface de G. Wicker, Issy-les-Moulineaux, LGDJ, 2019. 
monde en donnent une parfaite illustration. Dès la fin de l'année 2019, l'arrêt de l'économie chinoise a provoqué un ralentissement significatif de l'activité de nombreuses sociétés françaises. Ces sociétés sont certes indépendantes juridiquement, mais elles demeurent interdépendantes économiquement. Cela oblige à appréhender la question de la vulnérabilité dans le contexte d'une économie en réseau.

Dans une approche qui transcende les sous-disciplines du droit, cette étude tentera de démontrer que la vulnérabilité des sociétés est prise en compte par le droit positif, et d'en tirer les critères. Ceux-ci pourraient contribuer à la construction d'une théorie générale de la vulnérabilité, dont l'enjeu principal réside dans l'introduction d'une meilleure proportionnalité des mécanismes juridiques au regard de l'objectif de protection poursuivi. Sur le plan normatif, une meilleure identification des vulnérabilités pourrait réduire les excès de l'ordre public de protection dénoncés de façon récurrente par la doctrine. Sur le fond, la mise en lumière de la vulnérabilité à travers l'exemple des sociétés révélera la mouvance de cette notion sous l'effet des circonstances. Les sociétés sont davantage vulnérables depuis qu'elles sont devenues plus poreuses sous l'effet de la réorganisation de l'entreprise sous forme de réseau. C'est la raison pour laquelle cette étude s'articulera autour de la dichotomie société (organisation interne) / entreprise (environnement externe). Il conviendra donc d'analyser la vulnérabilité de la société à l'égard de ses propres organes, soit ses actionnaires et dirigeants (I), puis à l'égard de ses parties prenantes (II).

\section{La vulnérabilité de la société à l'égard de ses organes}

La société est naturellement propice aux conflits d'intérêts compte tenu du déséquilibre des rapports de force qu'elle abrite $^{4}$. En son sein, deux rapports se distinguent, celui de la société avec ses associés (A), puis celui de la société avec ses dirigeants (B).

\section{A. La vulnérabilité de la société à l'égard de ses associés}

Lorsqu'il s'agit de la relation entre une société et ses actionnaires, c'est le droit des sociétés qui sera interrogé sous le prisme de la vulnérabilité. Le droit des sociétés reconnaît depuis longtemps la vulnérabilité de la société vis-à-vis de ses actionnaires à travers des règles protectrices de l'intérêt social's. D'abord, la chambre commerciale de la Cour de cassation a développé une jurisprudence abondante relative à l'abus du droit de vote, qu'il soit commis par des majoritaires ${ }^{6}$, des minoritaires ${ }^{7}$ ou des associés égalitaires. Dans ce contexte, la vulnérabilité de la société découlera du risque de détournement d'un droit de sa finalité première, ce qui a pour conséquence l'atteinte à l'intérêt supérieur de la personne morale.

Depuis la loi $\mathrm{n}^{\circ}$ 2001-420 relative aux nouvelles régulations économiques du 15 mai 2001 (NRE), le droit des sociétés s'est davantage attaché à protéger l'actionnaire minoritaire vis-à-vis des majoritaires et des dirigeants, et in fine de la société. L'objectif de cette loi fut de renforcer les droits de ce dernier en lui conférant un véritable contre-pouvoir, encourageant par là même l'investissement financier ${ }^{8}$. Nombreuses sont les lois venues renforcer les droits de l'actionnaire minoritaire depuis, et jusqu'à la loi $\mathrm{n}^{\circ}$ 2019-486 du 22 mai 2019 relative à la croissance et la transformation des entreprises, dite loi PACTE9. À cet égard, la loi PACTE a transposé la directive $2017 / 828$ en vue de promouvoir l'engagement à long terme des actionnaires, ce qui a permis d'introduire une obligation de transparence des investisseurs institutionnels, des gestionnaires d'actifs et des agences de conseil en vote (proxy advisors).

Le renforcement légal des droits de l'actionnaire minoritaire a été mis à profit par les investisseurs institutionnels pour transformer la structure traditionnellement verticale du pouvoir, jugée plus favorable au majoritaire en raison de la supériorité de sa détention capitalistique. Autrement dit, l'horizontalisation de l'entreprise rend la société plus vulnérable face à l'activisme actionnarial déployé par les investisseurs institutionnels dans le cadre de l'ensemble

4. Les conflits d'intérêts dans le monde des affaires. Un Janus à combattre?, V. Magnier (dir), Paris, Presses universitaires de France (CEPRISCA), 2006; D. Schmidt, Les conflits d'intérêts dans la société anonyme, Paris, Joly (Pratique des affaires), 2004; A. Couret, «Les conflits d'intérêts dans la société anonyme, par Dominique Schmidt», Les petites affiches, nº 249, 15 décembre 1999, p. 12 sq. Plus récemment, la thèse de Valentin Baudouin s'attache à décrire l'entreprise comme un système d'intérêts communs encadré par un ordre public écologique (V. Baudouin, Études juridiques sur les petites et moyennes sociétés commerciales en transition écologique: l'entreprise sobre en contribution à une nouvelle approche de la RSE, thèse de doctorat en droit privé, université de Strasbourg, 2019, 340 p.). Au-delà du droit des sociétés, la thèse de J. Valiergue est particulièrement intéressante en ce qu'elle propose une grille de lecture des conflits d'intérêts en droit privé à travers le prisme de l'acte juridique (J. Valiergue, Les conflits d'intérêts en droit privé...).

5. B. Dupuis, La notion d'intérêt social, thèse de doctorat en droit, université Paris 13, 2001; T. Favario, L'intérêt de l'entreprise en droit privé français, thèse de doctorat en droit, université Lyon 3, 2004; A. Bennini, Le voile de l'intérêt social, Paris, Lextenso (LEJEP), 2013. Plus récemment: L'intérêt social dans la loi PACTE, É. Chevrier, E. Royer (dir.), Paris, Dalloz (Grand angle), 2019.

6. La Cour de cassation a défini l'abus de majorité dans son célèbre arrêt Piquard: Cass. com., 18 avril 1961, Recueil Dalloz, 1961, p. 166; La semaine juridique, édition générale, 1961, II, 12163, note D. Bastian.

7. La chambre commerciale de la Cour de cassation a consacré l'abus de minorité dans l'arrêt Six du 15 juillet 1992. Un an plus tard, la Cour a confirmé les conditions nécessaires à la qualification de l'abus de minorité dans l'arrêt Flandin du 9 mars 1993. Voir, en ce sens, Cass. com., 15 juillet 1992, Recueil Dalloz, 1993, p. 279; Cass. com., 9 mars 1993, Bulletin Joly Sociétés, 1993, p. 152, note P. Le Cannu.

8. D. Bureau, «La loi relative aux nouvelles régulations économiques. Aspects de droit des sociétés», Bulletin Joly sociétés, n 6, 2001 , p. 553 sq.; J. Dupichot, «La loi NRE et le droit des sociétés», Gazette du Palais, n 150 , 30 mai 2002, p. 3 sq.; Y. Guyon, «Les réformes apportées en droit des sociétés par la loi du 15 mai 2001 relative aux nouvelles régulations économiques", Revue des sociétés, 2001, p. 503 sq.

9. R. Arakélian, «Loi PACTE: aspects de droit des sociétés», Actualité juridique. Contrat, 2019, p. 272. 
du réseau. Deux techniques de déstructuration du pouvoir sont largement utilisées, cumulativement parfois, par les investisseurs institutionnels. Dans certains cas, cette déstructuration du pouvoir peut avoir pour conséquence la fragilisation de la société en ce qu'elle se trouvera tiraillée par des intérêts catégoriels.

La première technique de déstructuration du pouvoir réside dans le recours au groupe de sociétés qui peut être défini comme un ensemble de sociétés dénué de personnalité juridique, et dont la forme peut varier à l'infini. Chaque entité dispose d'une existence juridique propre, tout en étant sous le contrôle économique et stratégique de la société mère. Dans une structure classique, à savoir le groupe pyramidal, la société mère - actionnaire majoritaire - est contrôlaire, et ses filiales obéissent à une politique économique dictée par elle. Les filiales sont alors vulnérables en raison d'une centralisation du pouvoir décisionnel découlant de la notion de contrôle, tel qu'il est défini par l'article L. 233-3 du Code de commerce. Cette politique commune est d'ailleurs prise en compte aussi bien par le droit de la concurrence, qui légitime par exemple l'entente anticoncurrentielle entre filiales, que par le droit pénal des affaires qui consacre la notion d'intérêt du groupe comme fait justificatif de l'abus de bien social dans le célèbre arrêt de la chambre criminelle Rozenblum du 4 février $1985^{10}$.

À la lumière de ce qui précède, le recours au groupe de sociétés légitime la perpétration de pratiques interdites au détriment des filiales pourtant juridiquement indépendantes de leur mère. Ce contournement de la personnalité juridique des filiales génère leur propre vulnérabilité au profit de la société mère, et les notions d'intérêt du groupe et de politique du groupe en sont l'instrument. En effet, ces deux fictions juridiques viennent masquer la vulnérabilité des filiales au nom d'un intérêt supérieur. Pourtant, la fragilité des filiales est une réalité qui s'est accentuée avec la cohabitation entre actionnaires contrôlaires et investisseurs institutionnels, détenteurs d'un pouvoir financier quasi équivalent au pouvoir politique détenu par les majoritaires. Pour cette raison, l'Assemblée nationale envisage d'encadrer davantage l'activisme actionnarial à la suite d'un rapport d'information déposé par sa commission des finances le 2 octobre $2019^{11}$. Dans ce contexte, les filiales se trouvent assujetties à un pouvoir actionnarial décentralisé et diffus.

La seconde technique de déstructuration du pouvoir pyramidal des sociétés est l'entreprise en réseau. Dans ce contexte, une société peut être amenée à évoluer dans le cadre d'une entreprise organisée en réseau par ses actionnaires minoritaires, souvent investisseurs institutionnels. Ce réseau sera fondé sur un tissu contractuel renforcé qui a pour effet de mettre en œuvre un partenariat entre les firmes détenues par les mêmes investisseurs, de sorte à créer un ensemble homogène et efficient. Sur le plan de la gouvernance, ces détentions minoritaires constituent in fine un terrain global et propice à l'exercice de l'activisme actionnarial. Cette nouvelle forme d'organisation de l'entreprise en réseau génère indéniablement des vulnérabilités réciproques entre les sociétés qui en sont membres, en raison de leur interdépendance économique, contractuelle, outre la présence d'un pouvoir financier commun potentiellement source d'abus, comme tout actionnaire.

Il peut être soutenu que la société est vulnérable à l'égard de ses actionnaires en raison de conflits d'intérêts qui sous-tendent sa propre organisation. La fiction de la personnalité morale a longtemps dissimulé l'état de vulnérabilité intrinsèque de la société, consacrant l'intérêt supérieur de la personne morale comme la seule et unique boussole. Mais les nouvelles organisations de l'entreprise, comme le groupe et le réseau, qui transcendent la personnalité morale de la société, rappellent que cette dernière n'est qu'une organisation humaine, riche des forces et faiblesses des individus qui la composent, dont ses actionnaires et ses dirigeants.

\section{B. La vulnérabilité de la société à l'égard de ses dirigeants}

La vulnérabilité de la société vis-à-vis de l'action de son dirigeant de droit est reconnue implicitement par le législateur qui a élaboré un corpus de règles préventives et coercitives. Ces règles trouvent leur justification dans le risque d'abus du pouvoir de représentation et de direction du mandataire social. Dans le cadre d'un volet préventif, le législateur a notamment encadré les conventions conclues entre le dirigeant et la société dans les sociétés de capitaux et hybrides depuis la loi no 66-537 du 24 juillet 1966 relative aux sociétés commerciales. Certaines d'entre elles sont purement et simplement interdites ${ }^{12}$, comme l'octroi d'un découvert, d'un cautionnement ou d'un aval. D'autres sont réglementées ${ }^{13}$ en ce qu'elles supposent une appréciation in concreto, ainsi qu'un respect scrupuleux de la procédure

10. En matière d'abus de biens sociaux, la Cour de cassation conçoit qu'un tel acte puisse être perpétré dans l'intérêt du groupe de sociétés. Ce raisonnement légitime le «sacrifice économique» imposé à la filiale au nom d'une politique plus générale qui dépasserait ses frontières. Ce sacrifice économique doit être entendu de façon stricte, à la lumière de l'arrêt Rozenblum. La chambre commerciale a précisé que l'intérêt du groupe ne devait pas mettre en péril la viabilité d'une filiale, mais elle n'exclut pas le renoncement à son enrichissement au profit d'un intérêt supérieur (Cass. crim., 4 février 1985, La semaine juridique, édition générale, 1985, II, 20492, note A. Viandier).

11. P.-H. Conac, "L'Assemblée nationale envisage une amélioration des règles de transparence en matière d'activisme actionnarial», Revue des sociétés, 2020 , p. 63 sq.

12. Dans les sociétés anonymes, les conventions interdites sont visées à l'article L. 225-43 du Code de commerce.

13. Les conventions réglementées sont visées par plusieurs dispositions selon les formes sociales: art. L. 225-38 du Code de commerce pour les sociétés anonymes monistes; art. L. 225-86 du Code de commerce pour les sociétés anonymes dualistes; art. L. 227-10 du Code de commerce pour les sociétés par actions simplifiées; art. L. 223-19 du Code de commerce pour les sociétés à responsabilité limitée. 
légale de validation par les organes de la société qui varie selon les formes sociales. En réalité, ce mécanisme permet à la société de se doter d'un moyen de contrôle, et, in fine, de se prémunir d'éventuelles dérives de son dirigeant. La loi PACTE du 22 mai 2019 relative à la croissance et la transformation des entreprises a d'ailleurs renforcé ce dispositif en améliorant l'information des actionnaires en la matière ${ }^{14}$. Ces nouvelles dispositions sont salutaires même si le mécanisme reste perfectible puisque la notion même de convention réglementée n'est pas définie positivement par le législateur, ce qui génère un flou autour de leur qualification.

Dans le même esprit protectionniste, le droit français a introduit les principes américains de corporate governance, depuis l'entrée en vigueur de la loi relative aux nouvelles régulations économiques (NRE) du 15 mai $2001^{15}$. Ces principes se sont imposés comme le standard de la bonne gouvernance dans le monde. Parmi les obligations qui en découlent, on y trouve les devoirs de diligence, de loyauté, d'information des actionnaires et des parties prenantes. D'autres réformes sont venues consolider la protection de la société à l'égard de ses dirigeants, notamment par la limitation du cumul des mandats d'administrateur ${ }^{16}$.

Lorsque les règles de prévention ne suffisent plus, le dirigeant fautif est sanctionné. Sur le plan pénal, plusieurs infractions s'appliquent au dirigeant indélicat comme le délit d'abus de biens sociaux et le délit d'initié. Sur le plan civil, le dirigeant fautif peut être condamné pour faute de gestion à l'égard de la société ${ }^{17}$. Lorsque cette faute du dirigeant est à l'origine de la déconfiture de la société, le droit des procédures collectives parachèvera ce dispositif de protection de la société par la consécration de sanctions patrimoniales, professionnelles et pénales, faisant fi de l'écran de la personnalité morale en instaurant une action en responsabilité pour insuffisance d'actif à l'égard du dirigeant ${ }^{18}$.

À la lumière de ce qui précède, la vulnérabilité de la société à l'égard de son dirigeant de droit est inhérente au mandat de représentation et de direction du dirigeant qui lui confère un pouvoir susceptible de générer une situation d'abus ou d'incurie. En l'absence de mandat, le droit s'est saisi de la gestion de fait. Le dirigeant de fait est susceptible d'engager sa responsabilité civile délictuelle pour faute, et encourt les mêmes sanctions patrimoniales que le dirigeant de droit en cas de liquidation judiciaire. Dès lors, la vulnérabilité de la société vis-à-vis d'un dirigeant de fait découlera de son pouvoir d'influence sur la direction de celle-ci.

En conclusion, la vulnérabilité de la société à l'égard de ses organes (dirigeants et actionnaires) est intrinsèque à sa propre organisation. Elle est le revers de la dimension fictive de la personnalité morale, contrainte de se faire représenter et gérer par ses organes qui n'échappent pas à la réalité de toute organisation humaine naturellement tiraillée par des conflits d'intérêts. Mais la société a révélé davantage encore sa vulnérabilité à l'égard des tiers, depuis qu'elle est devenue une «boîte de verre ${ }^{19}$.

\section{La vulnérabilité de la société à l'égard des tiers}

La catégorie des tiers au contrat de sociétés est infinie. Se pose alors la difficulté de l'identification de ces tiers, dans un contexte économique où la structure sociétaire est le maillon d'une entreprise organisée en réseau. Une distinction peut être faite entre les tiers liés par un rapport contractuel avec la société (A), et ceux qui n'ont pas de lien juridique avec cette dernière mais demeurent des parties prenantes (B).

\section{A. Les vulnérabilités de la société à l'égard de ses cocontractants}

Les vulnérabilités de la société à l'égard de son environnement économique direct sont nombreuses, et les règles applicables à la concurrence, à la distribution et aux relations commerciales sont porteuses de mécanismes de protection des sociétés et des entreprises. S'inscrivent dans cet esprit plusieurs dispositions protectrices, dont la prohibition de l'abus de dépendance économique, les règles relatives aux délais de paiement ${ }^{20}$, les obligations précontractuelles du franchiseur à l'égard du franchisé ${ }^{21}$, la nécessité d'un préavis raisonnable en cas de rupture

14. J. Heinich, «Les conventions réglementées", Revue des sociétés, 2019, p. 619 sq.

15. N. Rontchevsky, «Le gouvernement d'entreprise à la française (Brèves observations sur le volet société de la loi NRE)», Recueil Dalloz, 20o1, p. 2578 sq.

16. Une personne physique ne peut exercer simultanément plus de cinq mandats d'administrateur de sociétés anonymes ayant leur siège sur le territoire français. Une dérogation existe pour le cas des sociétés contrôlées (art. L. 225-21 du Code de commerce). Ces principes ont été assouplis par deux textes successifs, les lois du 29 octobre 2002 et du $1^{\text {er }}$ août 2003 . Voir, en ce sens, J.-P. Dom, «Cumul des mandats: et si le remède s'avérait pire que mal?», Bulletin Joly Sociétés, n 10, 2002, p. 1095 sq.; B. Saintourens, «Le cumul des mandats sociaux au sein de la société anonyme après la loi du 29 octobre 2002 ", Revue des sociétés, 2003, p. 1 sq.

17. La faute de gestion est polymorphe, en ce qu'elle regroupe un nombre important de comportements qu'il est difficile de répertorier. Voir, en ce sens, E. Scholastique, Le devoir de diligence des administrateurs de sociétés. Droit français et anglais, Paris, LGDJ, 1998, p. 46 sq.; S. Di Meglio, «Les dirigeants et mandataires sociaux en droit français», Cahiers juridiques et fiscaux de l'exportation, ${ }^{\circ}$ 5, 1999, p. 1079 sq.

18. Art. L. 651-1 du Code de commerce.

19. M.-A. Frison-Roche, «Le modèle du marché», Archives de philosophie du droit, no 40, 1995, p. 286-313.

20. É. Chevrier, «Modernisation de l'économie: délais de paiement. Projet de loi de modernisation de l'économie, art. 6 et 6 bis nouveau », Recueil Dalloz, 2008, p. 1607.

21. D. Ferrier, «Nullité du contrat de franchise lorsque le franchiseur ne délivre pas l’information précontractuelle prévue par la loi », Recueil Dalloz, 1997, p. 55. 
totale ou partielle de relations commerciales établies ${ }^{22}$, et plus généralement la prohibition des pratiques restrictives de concurrence. S'ajoute aux règles nationales, une protection européenne générale et sectorielle ${ }^{23}$. Face à cette protection croissante de la partie faible dans les contrats conclus entre professionnels, la pratique a développé des contrats sui generis afin de retrouver une liberté contractuelle perdue. Les contrats de partenariats dont le but est d'échapper au cadre réglementé de la franchise en sont une bonne illustration. Mais, la consécration récente de la notion de déséquilibre significatif en droit des contrats est venue achever l'édifice de la protection de la partie cocontractante la plus faible économiquement dans les relations entre professionnels ${ }^{24}$.

Force est de constater que les relations contractuelles révèlent que l'état de vulnérabilité d'une société peut être provoqué par des facteurs extérieurs et contingents. Parce que cette situation de faiblesse est extériorisée, son caractère variable est plus visible. Il dépendra essentiellement de l'intensité des rapports de force en présence qui fluctuent sur toute la durée de l'exécution du contrat. Autrement dit, la partie faible n'est pas toujours celle que l'on croit, dans la mesure où la vulnérabilité peut changer de camp.

Mais lorsque les difficultés économiques d'une société se font jour, et qu'elles perturbent ses engagements contractuels, la vulnérabilité redevient intrinsèque à la société, même si sa cause peut lui être étrangère. Le droit des entreprises en difficulté intervient alors pour protéger la société de son état de fragilité financière, et l'ensemble des règles applicables dont l'arrêt des poursuites, l'arrêt du cours des intérêts, l'interdiction du paiement des créances antérieures, ont été pensées pour permettre son sauvetage lorsqu'elle est débitrice ${ }^{25}$. Paradoxalement, c'est au stade des procédures collectives qu'une société débitrice devient la moins vulnérable vis-à-vis de ses créanciers, en ce qu'elle est mise à l'abri de pressions extérieures de toute nature. Sa surprotection projette alors les créanciers dans l'incertitude, et même parfois dans un état de vulnérabilité économique.

Il convient toutefois de soustraire à ce dernier propos la procédure de conciliation. Parce qu'elle a été conçue dans l'esprit de prévenir les difficultés, la conciliation laisse un espace confiné à la liberté contractuelle ${ }^{26}$, ce qui peut amener une société débitrice à consentir à un accord confidentiel déséquilibré avec ses créanciers ${ }^{27}$. En cas de cessation des paiements consécutive à un tel accord, la société débitrice devra solliciter l'ouverture d'une procédure de redressement ou de liquidation judiciaire, ce qui le rendra caduc ${ }^{28}$.

Il apparaît, à travers le maillage des relations contractuelles auquel une société est partie, que cette dernière peut se trouver dans un état de faiblesse en raison d'un déséquilibre lié à une ou plusieurs relations contractuelles, dont la cause n'est pas toujours inhérente à la société ellemême. Les causes sont en effet plurielles. Elles sont parfois inhérentes à la qualité d'une partie au contrat, comme le franchisé ou le concessionnaire. Elles sont parfois liées au déséquilibre d'une ou plusieurs obligations prévues au contrat. Enfin, la cause de la vulnérabilité peut puiser sa source dans la surprotection juridique dont bénéficie l'autre partie.

En somme, le droit commun des contrats ainsi que certains contrats spéciaux organisent une protection de la partie faible y compris lorsqu'il s'agit d'une société, mais ce dispositif juridique demeure épars et hétérogène. Au-delà de son contenu, ses effets peuvent révéler un excès de protection générant par là même la vulnérabilité des cocontractants de la partie présumée faible. La construction d'une théorie générale de la vulnérabilité permettrait de s'extraire des clivages sous-disciplinaires, et des dichotomies artificielles telles que les notions de non-professionnels / consommateurs, professionnels, afin de privilégier la protection du cocontractant vulnérable dans le cadre d'une approche globale et unitaire.

\section{B. Les vulnérabilités de la société à l'égard de ses parties prenantes}

La société connaît de nouvelles fragilités en raison de pressions extérieures visant à intégrer les intérêts des parties prenantes dans la gouvernance des sociétés, en particulier lorsqu'elles sont financiarisées ${ }^{29}$. Mais pas seulement, car

22. Art. L. 442-1, II du Code de commerce (nouvelle numérotation issue de l'ordonnance $\mathrm{n}^{\circ}$ 2019-359 du 24 avril 2019 portant refonte du titre IV du livre IV du Code de commerce relatif à la transparence, aux pratiques restrictives de concurrence et aux autres pratiques prohibées) qui dispose: «II. Engage la responsabilité de son auteur et l'oblige à réparer le préjudice causé le fait, par toute personne exerçant des activités de production, de distribution ou de services de rompre brutalement, même partiellement, une relation commerciale établie, en l'absence d'un préavis écrit qui tienne compte notamment de la durée de la relation commerciale, en référence aux usages du commerce ou aux accords interprofessionnels. / En cas de litige entre les parties sur la durée du préavis, la responsabilité de l'auteur de la rupture ne peut être engagée du chef d'une durée insuffisante dès lors qu'il a respecté un préavis de dix-huit mois./Les dispositions du présent II ne font pas obstacle à la faculté de résiliation sans préavis, en cas d'inexécution par l'autre partie de ses obligations ou en cas de force majeure».

23. L. Idot, «La fin du premier mandat de la Commissaire Vestager marquée par un grand débat sur le rôle de la politique de concurrence», Revue trimestrielle de droit européen, 2019, p. 883 sq.

24. G. Chantepie, N. Sauphanor-Brouillaud, «Déséquilibre significatif», in Répertoire de droit commercial, Paris, Dalloz, mai 2019, actualisation février 2020, p. 170 sq.

25. F. Macorig-Venier, «Entreprise en difficulté: situation des créanciers», chap. 2 : «Restrictions aux droits des créanciers», in Répertoire des sociétés, Paris, Dalloz, mars 2013, actualisation janvier 2020, $\mathrm{n}^{\circ}$ 180-369.

26. La procédure de conciliation est régie par les articles L. 611-4 à L. 611-16 du Code de commerce

27. H. Monsèrié-Bon, «Décision d'homologation", in Répertoire de droit commercial, Paris, Dalloz, mars 2012, $\mathrm{n}^{\circ}$ 113-120.

28. Art. L. 611-12 du Code de commerce.

29. Les sociétés financiarisées sont celles qui font appel à un financement extérieur orienté vers les marchés, ce qui ne vise pas exclusivement les sociétés cotées. 
toutes les sociétés sont soumises à la vigilance de parties prenantes qu'il s'agisse de défenseurs de l'environnement, de la cause animale ou d'une alimentation plus saine ${ }^{30}$, de l'État qui défend la création et la sauvegarde de l'emploi, d'associations de consommateurs, d'associations de défense de l'environnement, d'investisseurs professionnels, leurs représentants et conseillers, des agences de notations, et plus largement d'épargnants. En somme, la liste des parties prenantes s'étend à l'infini, et recouvre toute personne ayant un intérêt légitime à faire valoir auprès de la société.

Dans ce contexte, la société ne peut ignorer ses parties prenantes qui sont une source importante d'apports en capitaux et en industrie. C'est précisément en raison de cette dépendance financière des sociétés à l'égard des marchés que certaines parties prenantes ont pu développer des actions militantes pour faire prévaloir leurs intérêts. En effet, la financiarisation des sociétés crée un terrain propice à l'activisme qui a pris une ampleur importante en France, particulièrement celui des investisseurs professionnels. Étant rappelé qu'un investisseur n'est pas nécessairement actionnaire, au moment où il exerce des pressions directes ou indirectes sur une société émettrice. Dans ce dernier cas, ses conseillers sont un vecteur essentiel de l'activisme des investisseurs.

La doctrine juridique désigne ces pressions extérieures exercées sur les sociétés d'activisme actionnarial bien que ses initiateurs ne soient pas toujours actionnaires. Ce vocable donne l'illusion d'un contenu homogène, alors que la réalité est plurielle. Pire, l'activisme actionnarial ne fait l'objet d'aucune définition légale ou jurisprudentielle. Il peut être décrit comme une action militante visant à déployer les moyens traditionnellement dévolus par le droit pour la défense des intérêts de ses initiateurs. Une telle action militante n'est pas fondamentalement contraire à l'intérêt social. Elle exprime une démocratie de marché dont l'intérêt est le déploiement de contre-pouvoir.

Concrètement, certaines actions militantes peuvent être déterminantes dans la nomination des dirigeants, la diminution de leurs rémunérations, ou le rétablissement de la transparence dans la gouvernance. En témoigne l'affaire des rémunérations excessives de M. Ghosn, ancien dirigeant du groupe Renault Nissan, dénoncées dès 2009 par Proxinvest, agence française de conseil en vote ${ }^{31}$. La rémunération de ce dernier au titre de ses fonctions chez Nissan n'avait pas été portée à la connaissance des actionnaires dans le rapport de gestion du groupe Renault, et aux investisseurs plus généralement, faisant fi de l'obligation de communiquer le montant des rémunérations et avantages de toute nature versés aux dirigeants par les sociétés contrôlées, dans le rapport de gestion ${ }^{32}$. Ce manque de transparence a été d'autant plus mal perçu par le marché que le groupe Renault avait perçu des aides de l'État français en 2009. La ténacité de Proxinvest à dénoncer ce défaut de transparence au fil de ses rapports annuels jusqu'en 2017 a sans doute participé à la sensibilisation de l'opinion publique, conduisant les actionnaires à rejeter la résolution relative à la rémunération de $M$. Ghosn lors de l'assemblée générale $2016^{33}$. Cela n'a cependant pas suffi à emporter la conviction du conseil d'administration de Renault qui a tout de même validé la rémunération de son dirigeant, alors même que l'État français en est membre.

$\mathrm{Au}$-delà des questions classiques relatives à la gouvernance stricto sensu, la menace d'un vote sanction peut planer sur certaines assemblées générales, afin de faire prévaloir une vision de la stratégie économique. À cet égard, la question de la responsabilité sociétale intéresse de plus en plus de conseillers et d'investisseurs ${ }^{34}$. Le 5 février 2020, GLASS LEWIS, l'une des agences de conseil en vote les plus influentes au monde, a publié sa politique pour la saison des assemblées générales 2020 , dans laquelle elle prône un vote sanction pour les entreprises jugées insuffisamment à l'écoute des préoccupations relatives à la responsabilité sociale.

Mais l'activisme devient de facto contre-productif, dès lors que l'action militante des parties prenantes vise à imposer des intérêts catégoriels au détriment de l'intérêt social. Cela peut être le cas à travers des prises de position courtes sur les marchés financiers qui se concrétisent juridiquement par une vente à découvert. Ce mécanisme permet à un vendeur, qui n'est pas propriétaire d'un titre au moment où il conclut l'accord de vente, de le céder dès lors qu'il s'engage à en être propriétaire au moment de sa livraison ${ }^{35}$. La finalité de la vente à découvert est d'atteindre une valorisation plus juste d'une entreprise, lorsqu'elle a été surévaluée. Pourtant, cette technique peut être utilisée à mauvais escient, provoquant la baisse du cours du titre au détriment de l'intérêt social, par le recours à des campagnes de communication agressives et non contradictoires.

En décembre 2019, l'Association française des entreprises privées (AFEP) a publié un rapport qui milite pour un encadrement légal de l'activisme actionnarial ${ }^{36}$ qu'elle

30. J. Igalens, P. Francoual, "Vigilance et parties prenantes», Revue Lamy droit des affaires, $\mathrm{n}^{\circ}{ }_{124} 1^{\mathrm{er}}$ mars 2017 , p. 1 sq.

31. Proxinvest, La rémunération des présidents exécutifs des sociétés cotées, Paris, Proxinvest, 2009, vol. I, p. 54-55; Proxinvest, La rémunération des dirigeants des sociétés du SFB 250, Paris, Proxinvest, 2011, p. 16-17; Proxinvest, La rémunération des dirigeants des sociétés du SFB 25o, Paris, Proxinvest, 2010, p. 103-104.

32. Obligation découlant de l'article L. 225-102-1 du Code de commerce. L'Autorité des marchés financiers (AMF) a considéré que le législateur visait à travers cette disposition l'ensemble des rémunérations perçues au sein des sociétés comprises dans le périmètre de consolidation de la société mère. Voir, en ce sens, Autorité des marchés financiers, «Rapport 2010 de l'AMF sur le gouvernement d'entreprise et la rémunération des dirigeants", 12 juillet 2010, p. 50.

33. Proxinvest, La rémunération des présidents exécutifs des sociétés françaises, Paris, Proxinvest, 2016, p. 22.

34. V. de Beaufort, «L'engagement actionnarial en France, vecteur de gouvernance pérenne?», Revue des sociétés, 2019 , p. 375 sq.

35. A. Tehrani, Les investisseurs protégés en droit financier, préface de T. Bonneau, Paris, LexisNexis (Bibliothèque de droit de l'entreprise), 2015 , p. 527 sq.

36. Association française des entreprises privées, «Activisme actionnarial», décembre 2019, rapport publié sur le site de l'AFEP: https://afep.com/ wp-content/uploads/2019/12/Activisme-actionnarial_Décembre-2019.pdf. 
définit comme tout comportement court-termiste porté par des «méthodes agressives » ${ }^{37}$. Cet appel a été entendu par le législateur, puisqu'un rapport d'information a été déposé par la commission des finances, de l'économie générale et du contrôle budgétaire de l'Assemblée nationale, le 2 octobre 2019, relativement à l'encadrement de l'activisme actionnarial. On y trouve, en premier lieu, une proposition de définition de l'activisme actionnarial. Il s'agit selon ce rapport de tout

[...] comportement adopté par un actionnaire, le plus souvent minoritaire, qui exige d'une société du changement, en faisant campagne, au-delà du dialogue bilatéral avec l'entreprise, auprès de sa direction, d'autres actionnaires et parfois publiquement ${ }^{38}$.

En second lieu, une série de treize propositions a été formulée afin d'encadrer l'activisme actionnarial exercé par les investisseurs et leurs conseillers. Parmi elles, la possibilité offerte à une société faisant l'objet d'une campagne publique la mettant en cause d'être destinataire des informations substantielles qui sont communiquées à ses actionnaires, par l'activiste à l'origine de la campagne; ou encore la possibilité offerte aux sociétés cotées de répondre de façon souple et rapide à des campagnes les mettant en cause publiquement, notamment par voie de presse. Le rapport de l'Assemblée nationale va jusqu'à préconiser un droit à communication en faveur des sociétés émettrices, lors de "périodes de silence» (quiet period), qui précéderaient la publication de résultats des investigations engagées par les activistes ${ }^{39}$.

Force est de constater que la définition proposée de l'activisme mérite d'être étendue, en ce qu'elle semble écarter l'action militante des conseillers en gouvernance et d'investisseurs n'ayant pas nécessairement la qualité d'actionnaire. Pourtant, le rapport d'information de l'Assemblée nationale souligne le rôle ambigu de certains acteurs, comme les agences de conseil en vote (proxy advisors), et reconnaît qu'il peut être utile de comprendre la logique de leurs interventions. À cet égard, la loi PACTE, a introduit quelques dispositions encadrant a minima cette activité de conseil ${ }^{40}$, dont l'obligation de transparence.

En réalité, il convient de s'interroger sur l'impact du pouvoir des conseillers en vote sur une gouvernance à la française qui, sur le plan culturel, a longtemps été très «verticale» et très «concentrée». Cette influence n'est en effet pas simplement opérationnelle. Elle peut également avoir un impact normatif et prescriptif sur les marchés en faisant évoluer les représentations sociales d'une «bonne» gouvernance. L'activité de conseil en vote peut donc être à l'origine de doctrines (plus ou moins neutres) susceptibles de modifier non seulement les représentations des différents acteurs du marché, mais aussi celles de la société civile dans son ensemble. Elle peut même, in fine, influencer le travail du législateur. Il s'agit donc d'une nouvelle forme d'activisme exercée par un acteur extérieur aux émetteurs concernés, alors que l'activisme traditionnel émanait plutôt de leurs actionnaires. L'idée même de la fiabilité qui soustend la mission de conseil en vote devrait être une valeur centrale dans les futurs débats législatifs, car leurs analyses dépassent largement la simple relation de confiance avec leurs mandants. Des analyses défaillantes seraient en effet susceptibles de provoquer des externalités négatives, et notamment une instabilité des marchés financiers qu'il est précisément nécessaire d'éviter.

En tout état de cause, cette réflexion autour de l'activisme des parties prenantes met en lumière les mécanismes générateurs de vulnérabilités des sociétés. En prenant appui sur la dépendance financière de certaines sociétés à l'égard des marchés, et en l'absence de cadre légal relatif à l'activisme en France, une partie prenante quelle qu'elle soit peut exercer des pressions en usant d'une communication asymétrique, doublée d'une asymétrie informationnelle sur le fond, ce qui revient à priver les sociétés en cause de contradictoire.

En conclusion, la vulnérabilité des sociétés est sous-tendue par la notion de partie faible lorsqu'elle est intrinsèque à la société elle-même. Mais l'état de vulnérabilité peut être provoqué par des facteurs extérieurs à la société. Dans ce cas, il sera le résultat d'une dissymétrie qui peut être décrite comme un déséquilibre entre la société et son environnement. Il portera sur une asymétrie informationnelle, économique, financière, voire d'une dépendance, ou d'une communication asymétrique privant la société d'un espace d'expression sur le marché. Dans tous les cas, la dissymétrie s'appréciera de façon in concreto, dans le cadre d'un rapport de force qui ne découlera pas nécessairement d'un lien juridique.

37. Ibid., p. 3. Pour une synthèse, voir P.-H. Conac, «L'AFEP n'apprécie pas l'activisme actionnarial “court-termiste” ", Revue des sociétés, 2020, p. 67 sq.

38. É. Woerth, B. Dirx, Rapport d'information déposé par la commission des finances, de l'économie générale et du contrôle budgétaire en conclusion des travaux d'une mission d'information relative à l'activisme actionnarial, $\mathrm{n}^{\circ} \mathbf{2 2 8 7}$, enregistré à la présidence de l'Assemblée nationale le 2 octobre 2019 , p. 13, en ligne: http://www.assemblee-nationale.fr/dyn/15/rapports/cion_fin/l15b2287_rapport-information.

39. Ibid., recommandation $\mathrm{n}^{\circ} 6$, p. 63 .

40. Art. L. 533-22-4 et L. 544-3 à L. 544-6 du Code monétaire et financier. 